\title{
A topology on lattice-ordered groups
}

\author{
Huanrong Wu, Qingguo Li*, Bin Yu \\ College of Mathematics and Econometrics, Hunan University, Changsha 410082, China.
}

Communicated by M. Bohner

\begin{abstract}
We introduce the concept of the strong-positive cone in a lattice-ordered group $(G, \leqslant, \cdot)$ and define the continuous latticeordered group. We also investigate the C-topology and bi-C-topology given on a lattice-ordered group. The main results obtained in this paper are as follows: $(1)(G, \leqslant, \cdot)$ is a continuous lattice-ordered group if and only if $(G, \leqslant)$ is a continuous poset; (2) for the bi-C-topology $\tau$ in a continuous lattice-ordered group $(G, \leqslant, \cdot),(G, \cdot, \tau)$ is a topological group and $(G, \leqslant, \tau)$ is a topological lattice.
\end{abstract}

Keywords: Lattice-ordered group, continuous, topology, topological group, topological lattice.

2010 MSC: 06F15, 06B35, 54H11.

(C)2018 All rights reserved.

\section{Introduction}

Lattice-ordered groups (also called l-groups) are an important class of partially ordered algebraic systems. The study of l-groups was initiated by Birkhoff $[9,12]$ and followed by many other researchers $[6,16,24]$. The monographs $[10,15]$ gave a systematic account of the basic theory of l-groups. Recently, l-groups have cropped up in many other areas of mathematics, for instance, in the theory of bézout domains [36, 37], ordered fields [7, 15], category theory [11], topology theory [5, 8, 21] and others [22].

In the 1960s, Scott introduced domain theory [31], aiming to provide mathematical models for computer program languages. Later, domain theory has got a great development in the areas of abstract theories of computation [28, 32], the semantics of programming languages [29, 34], logic and lambda calculus [3,33], and in other branches of mathematics [14, 17]. Now, domain theory is still hot in lambda calculus and programming languages [1, 2], stable domain theory [35, 38], category theory [20, 23], and has many applications in classical mathematics $[18,25,26,30]$.

Based on the characteristics of l-group and domain, we introduce continuous l-group, aiming to establish the application of domain theory in algebraic structure and also broaden the study space of l-group

\footnotetext{
*Corresponding author

Email addresses: xueerzaifei@126.com (Huanrong Wu), liqingguoli@aliyun.com (Qingguo Li), yubin20070119@sina.com (Bin $\mathrm{Yu}$ )
}

doi: $10.22436 /$ jnsa.011.05.10

Received: 2017-04-13 Revised: 2018-02-03 Accepted: 2018-03-01 
theory. As we all know that domain is a continuous dcpo and the approximation is the core of continuity. Therefore, in the course of the study of continuity, scholars pay more attention to the infinite case. And, by Proposition 2.1 given soon afterwards, we know that an l-group $G$ is an infinite set except that $G=\{e\}$. Thus, in the research of l-groups, scholars tend to study in the infinite set. Due to this consideration, we come up with an idea that we need to introduce continuity in l-group. In the field of mathematics, topology theory has always been the focus of our concern. Especially in recent years, topologies on l-group have received much more attention [8, 21, 22]. In this paper, the C-topology and bi-C-topology $\tau$ given on an l-group are studied. We also obtain the result that if $(G, \leqslant, \cdot)$ is a continuous l-group, then $(G, \cdot \tau)$ is a topological group and $(G, \leqslant, \tau)$ is a topological lattice.

The main task of this paper reads as follows. In Section 2, we shall first briefly introduce l-group and related basic facts. In Section 3, we introduce the strong-positive cone C and give the definition of the continuous l-group. And then some properties of the strong-positive cone and continuous l-group are studied. Finally, we investigate the C-topology and bi-C-topology defined on an l-group.

\section{Preliminaries}

In this section, we shall recall some notions and notations used in this paper. Let $X$ be a set and $\tau$ a family of subsets of $X$. Then $\tau$ is called a topology on $X$ if it satisfies: (1) both the empty set and $X$ are elements of $\tau$; (2) any union of elements of $\tau$ is an element of $\tau$; (3) any intersection of finitely many elements of $\tau$ is an element of $\tau$. If $\tau$ is a topology on $X$, then the pair $(X, \tau)$ is called a topological space. The members of $\tau$ are called open sets in $X$. A subset of $X$ is said to be closed if its complement is in $\tau$. A basis $\mathcal{B}$ for a topological space $(X, \tau)$ is a collection of open sets in $\tau$ such that every open set in $\tau$ can be written as a union of elements of $\mathcal{B}$. A function or map from one topological space to another is called continuous if the inverse image of any open set is open.

Let $\mathrm{P}$ be a set. An order (a partial order) on $\mathrm{P}$ is a binary relation $\leqslant$ on $\mathrm{P}$ such that, for all $x, y, z \in \mathrm{P}$, (1) $x \leqslant x,(2) x \leqslant y$ and $y \leqslant x$ imply $x=y$, (3) $x \leqslant y$ and $y \leqslant z$ imply $x \leqslant z$, are satisfied. A set $P$ equipped with an order relation $\leqslant$ is said to be a partially ordered set (poset). A poset $\mathrm{P}$ is called a lattice if the infimum and supermum of any pair of elements in $P$ exist. A poset $P$ is called a complete lattice if the infimum and supermum of any subset of $P$ exist. Let $D$ be a non-empty subset of a poset $P$. Then $D$ is said to be directed if, for every pair of elements $x, y \in D$, there exists $z \in D$ such that $x \leqslant z$ and $y \leqslant z$. A subset $A \subseteq P$ is called an upper set if for every $x \in A, y \in P$ with $x \leqslant y$, we have $y \in A$. Dually, a subset $A \subseteq P$ is called a lower set if for every $x \in A, y \in P$ with $x \geqslant y$, we have $y \in A$. A subset $A \subseteq P$ is called a filter if $A$ is a filtered upper set. For two elements $x, y$ in a poset $P, x \vee y, x \wedge y$ denote the supermum and infimum of $\{x, y\}$, respectively and for every subset $A$ of $P, \vee A, \wedge A$ denote the supermum and infimum of $A$, respectively. For $x \in P$, we write $\downarrow x=\{a \in P: a \leqslant x\}$ and $\uparrow x=\{a \in P: x \leqslant a\}$.

A lattice-ordered group $(G, \leqslant, \cdot)$ is a group $(G, \cdot)$ with a binary relation $\leqslant$ such that $(G, \leqslant)$ is a lattice and satisfies: $x \leqslant y$ implies $z \cdot x \leqslant z \cdot y$ and $x \cdot z \leqslant y \cdot z$ for any $x, y, z \in G$. A quantale $(S, \leqslant, \cdot)$ is a semigroup $(S, \cdot)$ with a binary relation $\leqslant \operatorname{such}$ that $(S, \leqslant)$ is a complete lattice and satisfies: $x \cdot\left(\bigvee_{i \in I} y_{i}\right)=\bigvee_{i \in I}\left(x \cdot y_{i}\right)$ and $\left(\bigvee_{i \in I} y_{i}\right) \cdot x=\bigvee_{i \in I}\left(y_{i} \cdot x\right)$. In the following paper, e represents the identity element of a group $G$. For any $x \in G, A, B \subseteq G$, we write $x \cdot A=\{x \cdot a: a \in A\}, A \cdot x=\{a \cdot x: a \in A\}, A \cdot B=\{a \cdot b: a \in A, b \in B\}$ and $A^{-1}=\left\{a^{-1}: a \in A\right\}$. Sometimes we will note an l-group $(G, \leqslant, \cdot)$ as $G$.

Proposition 2.1. Let $(\mathrm{G}, \leqslant, \cdot)$ be an l-group. Then $\mathrm{G}=\{\mathrm{e}\}$ or $|\mathrm{G}|$ is infinite, where $|\mathrm{G}|$ denotes the number of elements of $\mathrm{G}$.

Proof. It is obvious that $G=\{e\}$ is an l-group. Assume that $|G|>1$. Since $G$ is a lattice, there exist $a, b \in G$ such that $a<b$. Denote $c=a^{-1} \cdot b$ and so $c \in G, c>e$. Then we can construct an infinite subset $\left\{x_{n}: n \in \mathbf{N}\right\}$ of $G$. Let $x_{1}=c \cdot c, x_{2}=x_{1} \cdot x_{1}, \ldots, x_{n}=x_{n-1} \cdot x_{n-1}, \ldots$. Now we will show that for any $n \in \mathbf{N}, x_{n}>e$ and $x_{n}$ is an increasing sequence. Since $c>e$, we have $x_{n}=c^{2^{n}}>c>e$. Because $x_{n}=x_{n-1} \cdot x_{n-1}>x_{n-1}$, we have $\left\{x_{n}\right\}$ is an increasing sequence. Therefore $\left\{x_{n}: n \in \mathbf{N}\right\}$ is an infinite subset of $G$. Whence $G=\{e\}$ or $|G|$ is infinite. 
Definition $2.2([15,24])$. The element $x$ of an l-group $G$ is positive if $x \geqslant e$. The set of all positive elements of $\mathrm{G}$ is called the positive cone.

Definition $2.3([17,18])$. Let $P$ be a poset. For every $x, y \in P$, we say $x \ll y$ if, for every directed subset $D$ of $P$ for which $\bigvee D$ exists and $y \leqslant \bigvee D$, there exists $d \in D$ such that $x \leqslant d$.

$P$ is called a continuous poset, if for each $x \in P,\{y \in P: y \ll x\}$ is directed and $x=\bigvee\{y \in P: y \ll x\}$.

Proposition 2.4 ([24]). Let $G$ be an l-group. Then $(x \vee y)^{-1}=x^{-1} \wedge y^{-1}$ and $(x \wedge y)^{-1}=x^{-1} \vee y^{-1}$ hold for any $x, y \in G$.

Proposition $2.5([15,24])$. Let $\mathrm{G}$ be an l-group. If $\mathrm{D}$ is a directed subset of $\mathrm{G}$ with $\bigvee \mathrm{D}$ exists, then for any $\mathrm{a}, \mathrm{b} \in \mathrm{G}, \mathrm{a} \cdot \mathrm{D} \cdot \mathrm{b}=\{\mathrm{a} \cdot \mathrm{d} \cdot \mathrm{b}: \mathrm{d} \in \mathrm{D}\}$ is directed and $\bigvee(\mathrm{a} \cdot \mathrm{D} \cdot \mathrm{b})=\mathrm{a} \cdot(\bigvee \mathrm{D}) \cdot \mathrm{b}$.

Proposition 2.6 ([15]). Let $\mathrm{G}$ be an l-group.

(1) If $\mathrm{A}, \mathrm{B} \subseteq \mathrm{G}$ with $\bigvee \mathrm{A}, \bigvee \mathrm{B}$ exist, then $\bigvee(\mathrm{A} \cdot \mathrm{B})=\bigvee \mathrm{A} \cdot \bigvee \mathrm{B}$

(2) If $A, B \subseteq G$ with $\bigwedge A, \wedge B$ exist, then $\bigwedge(A \cdot B)=\bigwedge A \cdot \wedge B$.

Proposition $2.7([15,24])$. Let $\mathrm{G}$ be an l-group. If $\mathrm{D}$ is a directed subset of $\mathrm{G}$ with $\bigvee \mathrm{D}$ exists, then $\mathrm{D}^{-1}$ is a filtered set and $\bigwedge\left(\mathrm{D}^{-1}\right)=(\bigvee \mathrm{D})^{-1}$. Dually, if $\mathrm{F}$ is a filtered subset of $\mathrm{G}$ with $\wedge \mathrm{F}$ exists, then $\mathrm{F}^{-1}$ is a directed set and $\bigvee\left(\mathrm{F}^{-1}\right)=(\bigwedge \mathrm{F})^{-1}$.

Proposition 2.8 ([27]). Let $X$ be a set and $\mathcal{B}$ a family of subsets of $X . \mathcal{B}$ is a basis of a topology of $X$ if it satisfies:

(1) $\bigcup\{\mathrm{B}: \mathrm{B} \in \mathcal{B}\}=\mathrm{X}$;

(2) for any $\mathrm{B}_{1}, \mathrm{~B}_{2} \in \mathcal{B}$ and $\mathrm{x} \in \mathrm{B}_{1} \cap \mathrm{B}_{2}$, there exists $\mathrm{B}_{3} \in \mathcal{B}$ such that $\mathrm{x} \in \mathrm{B}_{3} \subseteq \mathrm{B}_{1} \cap \mathrm{B}_{2}$.

Definition $2.9([4]) \cdot(G, \cdot \tau(G))$ is called a topological group if it satisfies:

(1) $(G, \cdot)$ is a group and $\tau(G)$ is a topology on $G$;

(2) the map $f: G \times G \rightarrow G$ as mapping $(x, y)$ to $x \cdot y$ is continuous with respect to $\tau(G \times G)$ and $\tau(G)$;

(3) the map $g: G \rightarrow G$ as mapping $x$ to $x^{-1}$ is continuous with respect to $\tau(G)$.

Definition $2.10([19])$. ( $L, \leqslant, \tau(\mathrm{L}))$ is called a topological lattice if it satisfies:

(1) $(L, \leqslant)$ is a lattice and $\tau(L)$ is a topology on $L$;

(2) the map $f: L \times L \rightarrow L$ as mapping $(x, y)$ to $x \vee y$ is continuous with respect to $\tau(L \times L)$ and $\tau(L)$;

(3) the map $g: L \times L \rightarrow L$ as mapping $(x, y)$ to $x \wedge y$ is continuous with respect to $\tau(L \times L)$ and $\tau(L)$.

\section{Continuous lattice-ordered group}

In this section, we introduce the strong-positive cone which is a subset of the positive cone of an 1group. Furthermore, we study many properties of the strong-positive cone and give some good examples. Based on the strong-positive cone, we put forward the definition of continuous l-group and show that the direct product of two continuous l-groups is also a continuous l-group.

Definition 3.1. Let $G$ be an l-group with the positive cone $P$. An $x \in P$ is called a strong-positive element if for any directed subset $D$ of $G$ with $\bigvee D$ exists and $x=\bigvee D, D \cap P \neq \emptyset$ holds. The set of all strong-positive elements in $\mathrm{G}$ is called the strong-positive cone.

In domain theory, for elements $x, y$ in a poset $L$, we say $x \ll y$ if, for every directed subset $D$ of $L$ for which $\bigvee D$ exists and $y \leqslant \bigvee D$, there exists $d \in D$ such that $x \leqslant d$. So for an element $x$ in an l-group, $e \ll x$ means that for every directed subset $D$ of $G$ for which $\bigvee D$ exists and $x \leqslant \bigvee D$, there exists $d \in D$ such that $e \leqslant d$, that is $D \cap P \neq \emptyset$. Therefore, for an element $x$ in an l-group, $e \ll x$ implies that $x$ is a strong-positive element. However, if $x$ is a strong-positive element in an l-group, then we can't get that $e \ll x$ according to the definitions. In the following paper, you can see that " $e \ll x$ " and " $x$ is a strong-positive element" in an l-group are equivalent because of the particularity of l-groups. This will greatly simplify the definition of " $\ll$ " in l-group and continuous l-groups. For a deeper understanding of the strong-positive cone in an l-group, we give the following remark. 
Remark 3.2. Let $G$ be an l-group with the positive cone $P$ and the strong-positive cone $C . x \in P \backslash C$ if and only if $x \in P$ and there is a directed subset $D$ of $G \backslash P$ with $\bigvee D$ exists and $x=\bigvee D$.

\section{Example 3.3.}

(1) Let $(\mathbf{Z}, \leqslant,+)$ be the additive group with the standard order of integer number $\mathbf{Z} . \quad(\mathbf{Z}, \leqslant,+)$ is an l-group. The positive cone is $\{x \in \mathbf{Z}: x \geqslant 0\}$ and the strong-positive cone is $\{x \in \mathbf{Z}: x \geqslant 0\}$.

(2) Let $(\mathbf{R}, \leqslant,+)$ be the additive group with the standard order of $\mathbf{R}$. Obviously, $(\mathbf{R}, \leqslant,+)$ is an l-group. The positive cone is $\{x \in \mathbf{R}: x \geqslant 0\}$ and the strong-positive cone is $\{x \in \mathbf{R}: x>0\}$.

(3) Let $\left(\mathbf{Q}^{+}, \leqslant, \cdot\right)$ be the multiplicative group of positive rationals with the standard order. Then $\left(\mathbf{Q}^{+}, \leqslant, \cdot\right)$ is an l-group. The positive cone of $\left(\mathbf{Q}^{+}, \leqslant, \cdot\right)$ is $\left\{x \in \mathbf{Q}^{+}: x \geqslant 1\right\}$ and the strong-positive cone is $\left\{x \in \mathbf{Q}^{+}: x>1\right\}$.

(4) Let $\left(\mathbf{R}^{n},+\right)$ be the real $n$-space with the standard additive operator. The partial order is defined on $\left(\mathbf{R}^{n},+\right)$ by $\left(x_{1}, x_{2}, \ldots, x_{n}\right) \leqslant\left(y_{1}, y_{2}, \ldots, y_{n}\right)$ if $x_{i} \leqslant y_{i}$ for $1 \leqslant i \leqslant n$. The positive cone of $\left(\mathbf{R}^{n}, \leqslant,+\right)$ is $\left\{\left(x_{1}, x_{2}, \ldots, x_{n}\right): x_{i} \geqslant 0\right\}$ and the strong-positive cone is $\left\{\left(x_{1}, x_{2}, \ldots, x_{n}\right): x_{i}>0\right\}$.

It must be noted that, the strong-positive cone in an l-group may be empty, as you can see in the example below.

Example 3.4. Let $X$ be an infinite set, $G=F(X, \mathbf{R})$ be a set of all functions $f: X \rightarrow \mathbf{R}$ with the coordinate order: $f \leqslant g$ for $f, g \in G$ if $f(x) \leqslant g(x)$ for all $x \in X$. Then $G$ is an l-group under the partial order $\leqslant$ and the addition: $(f+g)(x)=f(x)+g(x)$. The positive cone $P$ of $G$ is $\{f \in G: f(x) \geqslant 0$ for every $x \in X\}$. The strong-positive cone $\mathrm{C}$ of $\mathrm{G}$ is empty.

Proof. Let $f \in P$. For any finite set $F \subseteq X$, define $f^{F} \in G$ with $f^{F}(x)=f(x)$ for $x \in F$ and $f^{F}(x)=-1$ for $x \in X \backslash F$. Consider $D=\left\{f^{F}: F \subseteq\right.$ fin $\left.X\right\}$. It is obvious that $D \subseteq G \backslash P$. For any $f^{F_{1}}, f^{F_{2}} \in D, f^{F_{1}} \leqslant f^{F_{1}} \cup F_{2}$ and $f^{F_{2}} \leqslant f^{F_{1}} \cup F_{2}$, so $D$ is directed and $\bigvee D=f$. By Remark 3.2, $f \in P \backslash C$. Therefore the strong-positive cone $C$ is empty.

In the following paper, we study the l-group in which the strong-positive cone is nonempty.

Proposition 3.5. Let $\mathrm{G}$ be an l-group with the positive cone $\mathrm{P}$ and the strong-positive cone $\mathrm{C}$. Then,

(1) $C$ is an upper set;

(2) $\mathrm{P} \cdot \mathrm{C} \subseteq \mathrm{C}, \mathrm{C} \cdot \mathrm{P} \subseteq \mathrm{C}$;

(3) for any $x \in G, x^{-1} \cdot C \cdot x \subseteq C$;

(4) for any $x \in G, x \cdot C=C \cdot x$ and for any $A \subseteq G, A \cdot C=C \cdot A$.

Proof.

(1) Let $x \in C, y \in G$ with $x \leqslant y$. It is obvious that $y \in P$. Let $D$ be a directed subset of $G$ with $\bigvee D$ exists and $y=\bigvee D$. By Proposition $2.5, D^{\prime}=x \cdot y^{-1} \cdot D$ is directed and $\bigvee D^{\prime}=x$. Since $x \in C$, we have $D^{\prime} \cap P \neq \emptyset$ by Definition 3.1. That is, there exists $d \in D$ such that $x \cdot y^{-1} \cdot d \in P$. So $x \cdot y^{-1} \cdot d \geqslant e$. Since $y \cdot x^{-1} \geqslant e$, we have $d=\left(y \cdot x^{-1}\right) \cdot\left(x \cdot y^{-1} \cdot d\right) \geqslant e$. So $D \cap P \neq \emptyset$ and by Definition $3.1, y \in C$. Therefore $C$ is an upper set.

(2) Let $x \in P, y \in C$. Then $x \cdot y \geqslant y$. By (1), $x \cdot y \in C$. Thus $P \cdot C \subseteq C$. Similarly, C $P \subseteq C$.

(3) Let $y \in C$. It is obvious that $x^{-1} \cdot y \cdot x \in P$. Suppose that $D$ is a directed subset of $G$ with $\bigvee D$ exists and $x^{-1} \cdot y \cdot x=\bigvee D$. By Proposition 2.5, $D^{\prime}=\left\{x \cdot d \cdot x^{-1}: d \in D\right\}$ is directed and $\bigvee D^{\prime}=y$. So there exists $d \in D$ such that $x \cdot d \cdot x^{-1} \in P$ by Definition 3.1. Hence $d \in P$ and $D \cap P \neq \emptyset$. Thus $x^{-1} \cdot y \cdot x \in C$ by Definition 3.1. Therefore $x^{-1} \cdot C \cdot x \in C$.

(4) Let $x \in G, a \in C$. By (3), $x \cdot a \cdot x^{-1} \in C$. So $x \cdot a=\left(x \cdot a \cdot x^{-1}\right) \cdot x \in C \cdot x$. Thus $x \cdot C \subseteq C \cdot x$. Similarly, $C \cdot x \subseteq x \cdot C$. Therefore $x \cdot C=C \cdot x$. Let $A \subseteq G$. Since for any $x \in G, x \cdot C=C \cdot x$, we have $A \cdot C=C \cdot A$. 
Proposition 3.6. Let $\mathrm{G}$ be an l-group with the strong-positive cone $\mathrm{C}$. For any $\mathrm{x}, \mathrm{y} \in \mathrm{G}$, if $\mathrm{x} \leqslant \mathrm{y}$, then $\mathrm{x} \cdot \mathrm{C}^{-1} \subseteq$ $\mathrm{y} \cdot \mathrm{C}^{-1}$ and $\mathrm{y} \cdot \mathrm{C} \subseteq \mathrm{x} \cdot \mathrm{C}$.

Proof. Let $x, y \in G$ with $x \leqslant y$. For any $z \in x \cdot C^{-1}$, we have $z^{-1} \cdot x \in C$. Since $x \leqslant y$, we have $z^{-1} \cdot x \leqslant z^{-1} \cdot y$. Thus $z^{-1} \cdot y \in C$ by Proposition 3.5 and $z \in y \cdot C^{-1}$. Therefore $x \cdot C^{-1} \subseteq y \cdot C^{-1}$. Since $x \leqslant y$, we have $y^{-1} \leqslant x^{-1}$. Hence $y^{-1} \cdot C^{-1} \subseteq x^{-1} \cdot C^{-1}$. Because $x^{-1} \cdot C^{-1}=(C \cdot x)^{-1}$, we have $(C \cdot y)^{-1} \subseteq(C \cdot x)^{-1}$ and $C \cdot y \subseteq C \cdot x$. Hence $y \cdot C \subseteq x \cdot C$ by Proposition 3.5 (4).

Proposition 3.7. Let $\mathrm{G}$ be an l-group with the positive cone $\mathrm{P}$ and the strong-positive cone $\mathrm{C}$. Then $\mathrm{C}$ is a filter.

Proof. By Proposition 3.5 (3), $C$ is an upper set. Let $x, y \in C$. We will show that $x \wedge y \in C$. Suppose that $D$ is a directed subset of $G$ with $\bigvee D$ exists and $\bigvee D=x \wedge y$. Consider $D_{1}=x \cdot(x \wedge y)^{-1} \cdot D$. By Proposition 2.5, $D_{1}$ is directed and $\bigvee D_{1}=x$. Since $x \in C$, we have $D_{1} \cap P \neq \emptyset$. That is, there exists $d_{1} \in D$ such that $x \cdot(x \wedge y)^{-1} \cdot d_{1} \in P$. Thus $d_{1} \geqslant(x \wedge y) \cdot x^{-1}$. Similarly, there exists $d_{2} \in D$ such that $d_{2} \geqslant(x \wedge y) \cdot y^{-1}$. Since $D$ is directed, there exists $d \in D$ such that $d \geqslant(x \wedge y) \cdot x^{-1}$ and $d \geqslant(x \wedge y) \cdot y^{-1}$. So $d \cdot(x \wedge y)^{-1} \geqslant x^{-1}$ and $d \cdot(x \wedge y)^{-1} \geqslant y^{-1}$. Hence $d \cdot(x \wedge y)^{-1} \geqslant x^{-1} \vee y^{-1}$. By Proposition 2.4, $x^{-1} \vee y^{-1}=(x \wedge y)^{-1}$. So $d \cdot(x \wedge y)^{-1} \geqslant(x \wedge y)^{-1}$ and $d \geqslant e$. Thus $D \cap P \neq \emptyset$ and $x \wedge y \in C$. Therefore $\mathrm{C}$ is a filter.

Definition 3.8. Let $\mathrm{G}$ be an l-group with the strong-positive cone $\mathrm{C}$. $\mathrm{G}$ is called a continuous l-group if, $\bigwedge C$ exists and $e=\bigwedge C$.

Comparing with the definition of continuous in domain theory, this definition is more simple and one can judge whether an l-group is continuous more quickly. Of course, by Theorem 3.14 given soon afterward, you can see these two definitions in an l-group are equivalent.

\section{Example 3.9.}

(1) Every totally ordered group is a continuous l-group.

(2) Let $\mathrm{G}$ be an l-group with the strong-positive cone $\mathrm{C}$. If $e \in C$, then $\mathrm{G}$ is a continuous l-group.

(3) Consider the Example $3.3(4)$. In $\left(\mathbf{R}^{n}, \leqslant,+\right)$, the strong-positive cone is $\left\{\left(x_{1}, x_{2}, \cdots, x_{n}\right): x_{i}>0\right\}$. Since $\bigwedge\left\{\left(x_{1}, x_{2}, \cdots, x_{n}\right): x_{i}>0\right.$ for $\left.1 \leqslant i \leqslant n\right\}=(0,0, \cdots, 0),\left(\mathbf{R}^{\mathfrak{n}}, \leqslant,+\right)$ is a continuous l-group.

(4) Consider the Example 3.4. In $(G, \leqslant,+)$, the strong-positive cone is empty. Since there does not exist the largest element in $(G, \leqslant,+)$, we have $\bigwedge \emptyset$ does not exist. Thus $(G, \leqslant,+)$ is not a continuous l-group.

Proposition 3.10. Let $\mathrm{G}$ be an l-group with the strong-positive cone $\mathrm{C}$. If there exists a set $\mathrm{F} \subseteq \mathrm{C}$ such that $\bigwedge \mathrm{F}=e$, then $\mathrm{G}$ is a continuous l-group.

Proof. It is obvious that $e$ is a lower bound of C. Suppose that $x$ is another lower bound of $C$. Since $F \subseteq C$, we have $e, x$ are lower bounds of $F . \wedge F=e$, so $x \leqslant e$. Thus $e$ is the largest lower bound of $C$. Hence $G$ is a continuous l-group by Definition 3.8.

Proposition 3.11. Let $\mathrm{G}$ be a continuous l-group with the strong-positive cone $\mathrm{C}$. Then for any $\mathrm{x} \in \mathrm{G}, \mathrm{x} \cdot \mathrm{C}^{-1}$ is directed and $\bigvee\left(x \cdot \mathrm{C}^{-1}\right)=\mathrm{x}$.

Proof. By Proposition 3.7, we have $C$ is filtered. Since $G$ is a continuous l-group, $\triangle C=e$. So $C^{-1}$ is directed and $\bigvee C^{-1}=e$ by Proposition 2.7. Hence for any $x \in G$, the set $x \cdot C^{-1}$ is directed and $\bigvee\left(x \cdot C^{-1}\right)=x$ by Proposition 2.5.

Proposition 3.12. Let $\mathrm{G}$ be a continuous l-group with the strong-positive cone $\mathrm{C}$. For any $\mathrm{x} \in \mathrm{C}$, if $\mathrm{D}$ is a directed subset of $\mathrm{G}$ with $\bigvee \mathrm{D}$ exists and $\mathrm{x}=\bigvee \mathrm{D}$, then $\mathrm{D} \cap \mathrm{C} \neq \emptyset$

Proof. Assume that $x \in C$ and $D$ is a directed subset of $G$ with $\bigvee D$ exists and $x=\bigvee D$. By Proposition 3.11, we have $C^{-1}$ is directed and $\bigvee C^{-1}=e$. So $D \cdot C^{-1}$ is directed and $\bigvee\left(D \cdot C^{-1}\right)=x$ by Proposition 2.6. Hence $\left(D \cdot C^{-1}\right) \cap P \neq \emptyset$ by Definition 3.1. That is, there exists $d \in D, a \in C$ such that $d \cdot a^{-1} \geqslant e$. Thus $d \geqslant a$. Since $C$ is an upper set by Proposition $3.5(1)$, we have $d \in C$ and $D \cap C \neq \emptyset$. 
Proposition 3.13. Let $\mathrm{G}$ be a continuous l-group with the strong-positive cone $\mathrm{C}$. Then $\mathrm{C} \cdot \mathrm{C}=\left\{\mathrm{x}_{1} \cdot \mathrm{x}_{2}: \mathrm{x}_{1} \in\right.$ $\left.\mathrm{C}, \mathrm{x}_{2} \in \mathrm{C}\right\}=\mathrm{C}$.

Proof. Since $\mathrm{C} \subseteq \mathrm{P}$, we have $\mathrm{C} \cdot \mathrm{C} \subseteq \mathrm{P} \cdot \mathrm{C} \subseteq \mathrm{C}$ by Proposition 3.5 (2). Thus $\mathrm{C} \cdot \mathrm{C} \subseteq \mathrm{C}$. For any $\mathrm{x} \in \mathrm{C}, \mathrm{x} \cdot \mathrm{C}^{-1}$ is directed and $\bigvee\left(x \cdot C^{-1}\right)=x$ by Proposition 3.11. Hence $\left(x \cdot C^{-1}\right) \cap C \neq \emptyset$ by Proposition 3.12. That is, there exists $a \in C$ such that $x \cdot a^{-1} \in C$. Since $x=\left(x \cdot a^{-1}\right) \cdot a \in C \cdot C$, we have $C \subseteq C \cdot C$. Therefore $\mathrm{C} \cdot \mathrm{C}=\mathrm{C}$.

Theorem 3.14. Let $(\mathrm{G}, \leqslant, \cdot)$ be an l-group. Then $(\mathrm{G}, \leqslant, \cdot)$ is a continuous l-group if and only if $(\mathrm{G}, \leqslant)$ is a continuous poset.

Proof. Let $\mathrm{P}$ be the positive cone of $\mathrm{G}, \mathrm{C}$ the strong-positive cone of $\mathrm{G}$. We will show that for any $x \in \mathrm{G}$, $\{y \in G: y \ll x\}=x \cdot C^{-1}$. Let $y \in G$ with $y \ll x$. Assume that $D$ is a directed subset of $G$ with $\bigvee D$ exists and $y^{-1} \cdot x=\bigvee D$. By Proposition 2.5, $y \cdot D$ is directed and $x=\bigvee(y \cdot D)$. Hence there exists $d \in D$ such that $y \leqslant y \cdot d$ by Definition 2.3. So $d \in P$ and $D \cap P \neq \emptyset$. Thus $y^{-1} \cdot x \in C$ by Definition 3.1. Because $y=x \cdot\left(y^{-1} \cdot x\right)^{-1}$, we have $y \in x \cdot C^{-1}$. Hence $\{y \in G: y \ll x\} \subseteq x \cdot C^{-1}$. Let $a \in C$. We will show that $x \cdot a^{-1} \ll x$. Assume that $D$ is a directed subset of $G$ with $\bigvee D$ exists and $x \leqslant \bigvee D$. By Proposition 2.5, $a \cdot(\bigvee D)^{-1} \cdot D$ is directed and $a=\bigvee\left(a \cdot(\bigvee D)^{-1} \cdot D\right)$. So $\left(a \cdot(\bigvee D)^{-1} \cdot D\right) \cap P \neq \emptyset$ by Definition 3.1. That is, there exists $d \in D$ such that $a \cdot(\bigvee D)^{-1} \cdot d \geqslant e$. Hence $d \geqslant(\bigvee D) \cdot a^{-1} \geqslant x \cdot a^{-1}$. We have $x \cdot a^{-1} \ll x$ by Definition 2.3. Thus $x \cdot C^{-1} \subseteq\{y \in G: y \ll x\}$. Therefore $\{y \in G: y \ll x\}=x \cdot C^{-1}$.

" $\Rightarrow "$ : Assume that $(G, \leqslant, \cdot)$ is a continuous l-group. Then for any $x \in G, x \cdot C^{-1}$ is a directed set and $\bigvee\left(x \cdot C^{-1}\right)=x$ by Proposition 3.11. Since $\{y \in G: y \ll x\}=x \cdot C^{-1}$, we have $\{y \in G: y \ll x\}$ is directed and $x=\bigvee\{y \in G: y \ll x\}$. Hence $(G, \leqslant)$ is a continuous poset by Definition 2.3.

" $\Leftarrow "$ : Since $(G, \leqslant)$ is a continuous poset and for any $x \in G,\{y \in G: y \ll x\}=x \cdot C^{-1}$, we have $x \cdot C^{-1}$ is directed and $x=\bigvee\left(x \cdot C^{-1}\right)$. Hence $e=\bigvee C^{-1}$ by Proposition 2.5 and $e=\bigwedge C$ by Proposition 2.7. Therefore $(G, \leqslant, \cdot)$ is a continuous l-group.

Proposition 3.15. Let $\left(\mathrm{G}_{1}, \leqslant 1, \cdot\right)$ and $\left(\mathrm{G}_{2}, \leqslant 2, *\right)$ be l-groups with the positive cones $\mathrm{P}_{1}$ and $\mathrm{P}_{2}$, respectively. Then the direct product $\left(\mathrm{G}_{1} \times \mathrm{G}_{2}, \leqslant, \star\right)$ with $\left(\mathrm{a}_{1}, \mathrm{~b}_{1}\right) \leqslant\left(\mathrm{a}_{2}, \mathrm{~b}_{2}\right)$ defined by $\mathrm{a}_{1} \leqslant 1 \mathrm{a}_{2}, \mathrm{~b}_{1} \leqslant 2 \mathrm{~b}_{2}$ and $\left(\mathrm{a}_{1}, \mathrm{~b}_{1}\right) \star\left(\mathrm{a}_{2}, \mathrm{~b}_{2}\right)=$ $\left(\mathrm{a}_{1} \cdot \mathrm{b}_{1}, \mathrm{a}_{2} * \mathrm{~b}_{2}\right)$ is an l-group and the positive cone of $\mathrm{G}_{1} \times \mathrm{G}_{2}$ is $\mathrm{P}_{1} \times \mathrm{P}_{2}$. If $\mathrm{C}_{1}, \mathrm{C}_{2}$ are the strong-positive cones of $\mathrm{G}_{1}, \mathrm{G}_{2}$, respectively, then in $\mathrm{G}_{1} \times \mathrm{G}_{2}$ the strong-positive cone $\mathrm{C}$ is $\mathrm{C}_{1} \times \mathrm{C}_{2}$.

Proof. It is obvious that $G_{1} \times G_{2}$ is an l-group and the positive cone is $P_{1} \times P_{2}$. Now we will show that the strong-positive cone $C=C_{1} \times C_{2}$. Let $\left(x_{1}, x_{2}\right) \in C$. Suppose that $D_{1}$ is a directed subset of $G_{1}$ with $x_{1}=\bigvee D_{1}$. Then $D_{1} \times\left\{x_{2}\right\}$ is a directed subset of $G_{1} \times G_{2}$. Since $\left(x_{1}, x_{2}\right) \in C$, there exists $d_{1} \in D_{1}$ such that $\left(d_{1}, x_{2}\right) \in P_{1} \times P_{2}$ which implies $d_{1} \in P_{1}$. Thus $x_{1} \in C_{1}$. Similarly, $x_{2} \in C_{2}$. Therefore $C \subseteq C_{1} \times C_{2}$. Let $\left(x_{1}, x_{2}\right) \in C_{1} \times C_{2}$. Suppose that $D_{1} \times D_{2}$ is a directed subset of $G_{1} \times G_{2}$ with $\left(x_{1}, x_{2}\right)=\bigvee\left(D_{1} \times D_{2}\right)$. So $D_{1}$ is a directed subset of $G_{1}$ with $x_{1}=\bigvee D_{1}$ and $D_{2}$ is a directed subset of $G_{2}$ with $x_{2}=\bigvee D_{2}$. Since $\left(x_{1}, x_{2}\right) \in C_{1} \times C_{2}, D_{1} \cap P_{1} \neq \emptyset$ and $D_{2} \cap P_{2} \neq \emptyset$. So $\left(D_{1} \times D_{2}\right) \cap\left(P_{1} \times P_{2}\right) \neq \emptyset$. Thus $\left(x_{1}, x_{2}\right) \in C$ and $\mathrm{C}_{1} \times \mathrm{C}_{2} \subseteq \mathrm{C}$. Therefore $\mathrm{C}=\mathrm{C}_{1} \times \mathrm{C}_{2}$.

Theorem 3.16. Let $\left(\mathrm{G}_{1}, \leqslant 1, \cdot\right)$ and $\left(\mathrm{G}_{2}, \leqslant 2, *\right)$ be continuous l-groups. Then the direct product $\mathrm{G}_{1} \times \mathrm{G}_{2}$ is a continuous l-group.

Proof. This result can be proved by Proposition 3.15.

\section{A topology on lattice-ordered groups}

In this section, the C-topology and bi-C-topology $\tau$ given on an l-group are studied. We investigate some properties of these topologies and obtain that for a continuous l-group $(G, \leqslant, \cdot),(G, \cdot \tau)$ is a topological group and $(G, \leqslant, \tau)$ is a topological lattice. 
Proposition 4.1. Let $\mathrm{G}$ be an l-group with the strong-positive cone $\mathrm{C}$. The sets of the form $\mathrm{x} \cdot \mathrm{C}, \mathrm{x} \in \mathrm{G}$, constitute a basis of a topology on $\mathrm{G}$ (we call this topology the C-topology and denote it by $\beta(\mathrm{G})$ ).

Proof. Let $x \in G$ and $y \in x \cdot C^{-1}$. Then there exists $a \in C$ such that $y=x \cdot a^{-1}$. Thus $x=y \cdot a \in y \cdot C \subseteq$ $\bigcup\{x \cdot C: x \in G\}$. Therefore $G \subseteq \bigcup\{x \cdot C: x \in G\} \subseteq G$ and $G=\bigcup\{x \cdot C: x \in G\}$. Let $x, y \in G$. Since $G$ is an l-group, $x \vee y$ exists. By Proposition 3.6, $(x \vee y) \cdot C \subseteq x \cdot C$ and $(x \vee y) \cdot C \subseteq y \cdot C$. Hence $\{x \cdot C: x \in G\}$ constitutes a basis of a topology on $\mathrm{G}$ by Proposition 2.8 .

\section{Example 4.2.}

(1) Consider Example $3.3(1)$. In $(\mathbf{Z}, \leqslant,+)$, the basis of the C-topology is $\{[x,+\infty): x \in \mathbf{Z}\}$.

(2) Consider Example 3.3 (4). In $\left(\mathbf{R}^{n}, \leqslant,+\right)$, the basis of the C-topology is $\left\{\left(\uparrow\left(x_{1}, x_{2}, \ldots, x_{n}\right)\right) \backslash\left(x_{1}, x_{2}, \ldots\right.\right.$, $\left.\left.x_{n}\right): x_{i(1 \leqslant i \leqslant n)} \in \mathbf{R}\right\}$.

Proposition 4.3. Let $\mathrm{G}$ be an l-group with the strong-positive cone $\mathrm{C}$.

(1) $\mathrm{U} \in \beta(\mathrm{G})$ if and only if $\mathrm{U}=\mathrm{U} \cdot \mathrm{C}$.

(2) If $U \in \beta(G)$, then $x \cdot U \in \beta(G)$.

(3) If $A$ is a closed set in $\beta(G)$, then for every $x \in G, x \cdot A$ is also a closed set.

Proof.

(1) " $\Rightarrow "$ " : Let $U \in \beta(G)$. Then $U=\bigcup_{x \in A \subseteq G} x \cdot C=A \cdot C$ by Proposition 4.1. So $U \cdot C=A \cdot C \cdot C$. By Proposition 3.13, $\mathrm{C} \cdot \mathrm{C}=\mathrm{C}$. Hence $\mathrm{U} \cdot \mathrm{C}=\bar{A} \cdot \mathrm{C}$. Therefore $\mathrm{U}=\mathrm{U} \cdot \mathrm{C}$.

" $\Leftarrow "$ : Since $\mathrm{U}=\mathrm{U} \cdot \mathrm{C}=\bigcup_{\mathrm{u} \in \mathrm{U}} \mathrm{u} \cdot \mathrm{C}$, we have $\mathrm{U} \in \beta(\mathrm{G})$ by Proposition 4.1.

(2) By (1), $U=U \cdot C$. Thus $x \cdot U=x \cdot U \cdot C=(x \cdot U) \cdot C$. Hence $x \cdot U$ is also an open set by (1).

(3) We will show that $x \cdot(G \backslash A)=G \backslash(x \cdot A)$. Let $a \in G \backslash A$ which implies $a \notin A$. So $x \cdot a \notin x \cdot A$, that is $x \cdot a \in G \backslash(x \cdot A)$. Thus $x \cdot(G \backslash A) \subseteq G \backslash(x \cdot A)$. Let $a \in G \backslash(x \cdot A)$ which implies $a \notin x \cdot A$. So $x^{-1} \cdot a \notin A$, that is $x^{-1} \cdot a \in G \backslash A$. Thus $a=x \cdot\left(x^{-1} \cdot a\right) \in x \cdot(G \backslash A)$ and $G \backslash(x \cdot A) \subseteq x \cdot(G \backslash A)$. Therefore $x \cdot(G \backslash A)=G \backslash(x \cdot A)$. Since $A$ is a closed set, we have $G \backslash A$ is an open set. Thus $x \cdot(G \backslash A)$ is also an open set by (2). Hence $G \backslash(x \cdot A)$ is an open set and $x \cdot A$ is a closed set.

Definition 4.4. A quantale $G$ is called completely distributive if for any family $\left\{x_{j, k}: j \in J, k \in K(j)\right\}$ in $G$ the identity

$$
\bigwedge_{j \in J} \bigvee_{k \in K(j)} x_{j, k}=\bigvee_{f \in M} \bigwedge_{j \in J} x_{j, f(j)}
$$

holds, where $M$ is the set of choice functions defined on $J$ with values $f(j) \in K(\mathfrak{j})$.

Proposition 4.5 ([13]). Let $\mathrm{P}$ be a non-empty poset. $\mathrm{P}$ is a complete lattice if and only if $\mathrm{P}$ has a least element and $\checkmark \mathrm{S}$ exists in $\mathrm{P}$ for any non-empty subset $\mathrm{S}$ of $\mathrm{P}$.

Proposition 4.6. Let $(\mathrm{G}, \leqslant, \cdot)$ be an l-group with the strong-positive cone $\mathrm{C} .(\beta(\mathrm{G}), \subseteq, \cdot)$ is a completely distributive quantale, where $\mathrm{U}_{1} \cdot \mathrm{U}_{2}=\left\{\mathrm{u}_{1} \cdot \mathrm{u}_{2}: \mathrm{u}_{1} \in \mathrm{U}_{1}, \mathrm{u}_{2} \in \mathrm{U}_{2}\right\}$ for any $\mathrm{U}_{1}, \mathrm{U}_{2} \in \beta(\mathrm{G})$.

Proof. By Proposition 4.5 , we have $(\beta(\mathrm{G}), \subseteq)$ is a complete lattice in which for $\mathrm{U}_{\mathrm{i}(\mathrm{i} \in \mathrm{I})} \in \beta(\mathrm{G})$

$$
\bigvee_{i \in I} u_{i}=\bigcup_{i \in I} u_{i}, \bigwedge_{i \in I} u_{i}=\bigcup\left\{v \in \beta(G): v \subseteq \bigcap_{i \in I} u_{i}\right\}
$$

For any $\mathrm{U}_{1}, \mathrm{U}_{2} \in \beta(\mathrm{G}), \mathrm{U}_{1} \cdot \mathrm{U}_{2}=\mathrm{U}_{1} \cdot \mathrm{C} \cdot \mathrm{U}_{2} \cdot \mathrm{C}$. By Proposition $3.5(4), \mathrm{C} \cdot \mathrm{U}_{2}=\mathrm{U}_{2} \cdot \mathrm{C}$. So $\mathrm{U}_{1} \cdot \mathrm{U}_{2}=$ $\mathrm{U}_{1} \cdot \mathrm{U}_{2} \cdot \mathrm{C} \cdot \mathrm{C}$. By Proposition 3.13, $\mathrm{C} \cdot \mathrm{C}=\mathrm{C}$. So $\mathrm{U}_{1} \cdot \mathrm{U}_{2}=\mathrm{U}_{1} \cdot \mathrm{U}_{2} \cdot \mathrm{C}$ and by Proposition $4.3(1)$, $\mathrm{U}_{1} \cdot \mathrm{U}_{2} \in \beta(\mathrm{G})$. Thus $(\beta(\mathrm{G}), \cdot)$ is a semigroup. Let $A, \mathrm{~B}_{i(i \in I)} \in \beta(G)$. We have $A \cdot\left(\bigvee_{i \in I} B_{i}\right)=A \cdot\left(\bigcup_{i \in I} B_{i}\right)=$ 
$A \cdot\left(\bigcup_{i \in I} B_{i}\right) \cdot C=\bigcup_{i \in I}\left(A \cdot B_{i} \cdot C\right)=\bigcup_{i \in I}\left(A \cdot B_{i}\right)=\bigvee_{i \in I}\left(A \cdot B_{i}\right)$. Thus $A \cdot\left(\bigvee_{i \in I} B_{i}\right)=\bigvee_{i \in I}\left(A \cdot B_{i}\right)$. Similarly, $\left(\bigvee_{i \in I} B_{i}\right) \cdot A=\bigvee_{i \in I}\left(B_{i} \cdot A\right)$. Therefore $(\beta(G), \subseteq, \cdot)$ is a quantale.

Then we will show that $G$ is completely distributive. For any family $\left\{U_{j, k}: j \in J, k \in K(j)\right\}$ in $G$, the inequality $\bigwedge_{j \in J} \bigvee_{k \in K(j)} U_{j, k} \geqslant \bigvee_{f \in M} \bigwedge_{j \in J} U_{j, f(j)}$ holds in any complete lattice. So we only need to show

that $\bigwedge_{j \in J} \bigvee_{k \in K(j)} u_{j, k} \leqslant \bigvee_{f \in M} \bigwedge_{j \in J} U_{j, f(j)}$, i.e., $\bigwedge_{j \in J} \bigvee_{k \in K(j)} u_{j, k} \subseteq \underset{f \in M}{\bigvee} \bigwedge_{j \in J} u_{j, f(j)}$. For any $x \in \bigwedge_{j \in J} \bigvee_{k \in K(j)} u_{j, k}$ there exists $y \in \bigwedge \bigcup_{j \in k} u_{j, k}$ such that $x \in y \cdot C$ by Proposition 4.3 (1). Since $\bigwedge \bigvee U_{j, k}=\bigcup\{V \in$ $\left.\beta(G): V \subseteq \bigcap_{j \in J}\left(\bigvee_{k \in K(j)} u_{j, k}\right)\right\}$, we have $y \in \bigvee_{k \in K(j)} u_{j, k}$ for any $j \in J$. Because $\underset{k \in K(j)}{\bigvee} u_{j, k}=\bigcup_{k \in K(j)} u_{j, k}$, we conclude that for any $j \in J$ there exists $k_{j} \in K(j)$ such that $y \in U_{j, k_{j}}$. We denote this $k_{j}$ by $f(j)$. By these choices we have found a function $f \in M$ such that $y \in \bigcap_{j \in J} u_{j, f(j)}$. Since $u_{j, f(j)} \in \beta(G)$, we have $y \cdot C \subseteq \mathrm{u}_{j, f(j)}$ for any $j \in J$ by Proposition 4.3 (1). Thus $y \cdot C \subseteq \bigcap_{j \in J} \mathrm{u}_{j, f(j)}$. Because $y \cdot C \in \beta(G)$, we have $y \cdot C \subseteq \bigwedge_{j \in J} u_{j, f(j)}=\bigcup\left\{V \in \beta(G): V \subseteq \bigcap_{j \in J} u_{j, f(j)}\right\}$. Thus $y \cdot C \subseteq \bigvee_{f \in M} \bigwedge_{j \in J} U_{j, f(j)}$ and $x \in \bigvee_{f \in M} \bigwedge_{j \in J} U_{j, f(j)}$. Hence $\bigwedge_{j \in J}^{j \in J} \bigvee_{k \in K(j)} u_{j, k} \subseteq \bigvee_{f \in M} \bigwedge_{j \in J} u_{j, f(j)}$. Therefore $\bigwedge_{j \in J} \bigvee_{k \in K(j)} u_{j, k}=\bigvee_{f \in M} \bigwedge_{j \in J}^{f \in J} u_{j, f(j)}$.

From the above discussion, we have $(\beta(G), \subseteq, \cdot)$ is a completely distributive quantale.

Definition 4.7 ([18]). Let $\mathrm{P}$ be a poset. We say that $\mathrm{U} \subseteq \mathrm{P}$ is Scott-open (open for the Scott topology of $\mathrm{P}$ ) if it satisfies the following two conditions:

(1) $U$ is an upper set;

(2) $\bigvee D \in U$ implies $D \cap U \neq \emptyset$, for any directed subset $D$ of $P$ with $\bigvee D$ exists.

Lemma 4.8 ([18]). Let $\mathrm{P}$ be a poset. For any $\mathrm{x} \in \mathrm{P}, \downarrow x$ is closed in the Scott topology of $\mathrm{P}$.

Theorem 4.9. Let $\mathrm{G}$ be an l-group with the strong-positive cone $\mathrm{C}$. Then the following conditions are equivalent:

(1) $\downarrow$ e is closed in the C-topology;

(2) $\mathrm{G}$ is a continuous l-group;

(3) the C-topology $\beta(\mathrm{G})$ is equivalent to the Scott topology of $(\mathrm{G}, \leqslant)$.

Proof.

(1) $\Rightarrow$ (2) Since $C \subseteq P, e$ is a lower bound of $C$. Assume that $x \in G$ is another lower bound of $C$. Then we will show that $x \leqslant e$. Suppose $x \nless e$. By (1), $G \backslash \downarrow e$ is open which contains $x$. So there exists $y \in G \backslash \downarrow e$ such that $x \in y \cdot C$ by Proposition $4.3(1)$. Thus $y^{-1} \cdot x \in C$. Since $x$ is a lower bound of $C$, we have $x \leqslant y^{-1} \cdot x$. Hence $y \leqslant e$ which is in contradiction with $y \in G \backslash \downarrow e$. So $x \leqslant e$ and $e$ is the largest lower bound of $C$. Therefore $\mathrm{G}$ is a continuous l-group.

(2) $\Rightarrow$ (3) Let $\mathrm{U} \in \beta(\mathrm{G})$ and $x \in \mathrm{U}, \mathrm{y} \in \mathrm{G}$ with $x \leqslant y$. Then there exist $u \in U, a \in C$ such that $x=u \cdot a$ by Proposition 4.3 (1). Since $x=u \cdot a \leqslant y$, we have $a \leqslant u^{-1} \cdot y$. So $u^{-1} \cdot y \in C$ by Proposition 3.5 (1). Thus $y=u \cdot\left(u^{-1} \cdot y\right) \in u \cdot C \subseteq U$. Hence $y \in U$ and $U$ is an upper set. Assume that $D$ is a directed subset of $G$ with $\bigvee D$ exists and $\bigvee D \in U$. Then there exists $u \in U$ such that $\bigvee D \in u \cdot C$ by Proposition 4.3 (1). So $u^{-1} \cdot(\bigvee D) \in C$. By Proposition 2.5, $u^{-1} \cdot D$ is directed and $\bigvee\left(u^{-1} \cdot D\right)=u^{-1} \cdot(\bigvee D) \in C$. Hence $\left(u^{-1} \cdot D\right) \cap C \neq \emptyset$ by Proposition 3.12. That is, there exists $d \in D$ such that $u^{-1} \cdot d \in C$. Thus $d \in u \cdot C \subseteq U$ and $\mathrm{D} \cap \mathrm{U} \neq \emptyset$. Therefore $\mathrm{U}$ is Scott open.

Conversely, let $U$ be Scott open. Suppose that $x \in U, a \in C$. Then $x \cdot a \geqslant x$ and $x \cdot a \in U$ because $U$ is an upper set. Thus $\mathrm{U} \cdot \mathrm{C} \subseteq \mathrm{U}$. By Proposition 3.11, for any $x \in G, x \cdot \mathrm{C}^{-1}$ is directed and $\bigvee\left(x \cdot \mathrm{C}^{-1}\right)=x$. Hence $\left(x \cdot c^{-1}\right) \cap U \neq \emptyset$ by Proposition 4.7. That is, there exists $c \in C$ such that $x \cdot c^{-1} \in U$. So $x=\left(x \cdot c^{-1}\right) \cdot c \in U \cdot c \subseteq U \cdot C$. Thus $U \subseteq U \cdot C$. Hence $\mathrm{U}=\mathrm{U} \cdot \mathrm{C}$ and $\mathrm{U} \in \beta(\mathrm{G})$ by Proposition 4.3 (1).

From the above discussion, we conclude that if $(G, \leqslant, \cdot)$ is a continuous l-group, the C-topology $\beta(G)$ is equivalent to the Scott topology of $(G, \leqslant)$. 
(3) $\Rightarrow$ (1) This result can be proved by Lemma 4.8 .

Lemma 4.10 ([27]). Let $(X, \tau)$ be a topological space and $A \subseteq X . A \in \tau$ if and only if for any $x \in A$, there exists $\mathrm{U} \in \tau$ such that $\mathrm{x} \in \mathrm{U} \subseteq \mathrm{A}$.

Proposition 4.11. Let $\mathrm{G}_{1}, \mathrm{G}_{2}$ be continuous l-groups with the strong-positive cones $\mathrm{C}_{1}, \mathrm{C}_{2}$, respectively. A function $f: G_{1} \rightarrow G_{2}$ is continuous with respect to the C-topologies of $G_{1}$ and $G_{2}$ if and only if for any $x \in G_{1}, f(x)=$ $\bigvee\left\{f(w): w \in x \cdot C_{1}{ }^{-1}\right\}$

Proof.

" $\Rightarrow "$ : Firstly, we will show that $f(x)$ is an upper bound of $\left\{f(w): w \in x \cdot C_{1}{ }^{-1}\right\}$. Assume that there exists $w_{0} \in x \cdot C_{1}^{-1}$ such that $f\left(w_{0}\right) \nless f(x)$. By Proposition 4.9 and Proposition $4.3(3), f(x) \cdot \downarrow e=\downarrow f(x)$ is closed and so, $V=G_{2} \backslash \downarrow f(x)$ is an open set and $f\left(w_{0}\right) \in V, f(x) \notin V$. Since $f$ is continuous, $U=f^{-1}(V)$ is an open set and $w_{0} \in \mathrm{U}, \mathrm{x} \notin \mathrm{U}$. Hence $w_{0} \cdot \mathrm{C}_{1} \subseteq \mathrm{U}$ by Proposition 4.3 (1). We have $x \in w_{0} \cdot \mathrm{C}_{1} \subseteq \mathrm{U}$. This contradicts $x \notin \mathrm{U}$. Thus for any $w \in x \cdot \mathrm{C}_{1}{ }^{-1}, \mathrm{f}(w) \leqslant \mathrm{f}(\mathrm{x})$.

Then we will show that $f(x)$ is the least upper bound of $\left\{f(w): w \in x \cdot C_{1}{ }^{-1}\right\}$. Suppose that $t \in G_{2}$ is another upper bound of $\left\{f(w): w \in x \cdot C_{1}^{-1}\right\}$. We only need to show that $f(x) \leqslant t$. Assume that $f(x) \nless t$. Then $G_{2} \backslash \downarrow t$ is an open set and $f(x) \in G_{2} \backslash \downarrow t$. Since $f$ is continuous, $f^{-1}\left(G_{2} \backslash \downarrow t\right)$ is an open set and $x \in f^{-1}\left(G_{2} \backslash \downarrow t\right)$. Hence there exists $d \in f^{-1}\left(G_{2} \backslash \downarrow t\right)$ such that $x \in d \cdot C_{1}$ by Proposition 4.3 (1). We have $f(d) \in G_{2} \backslash \downarrow t$ and $d \in x \cdot C_{1}{ }^{-1}$. Thus $f(d) \& t$. This contradicts that $t$ is an upper bound of $\left\{\mathrm{f}(w): w \in x \cdot C_{1}{ }^{-1}\right\}$. Therefore $\mathrm{f}(\mathrm{x})=\bigvee\left\{f(w): w \in x \cdot C_{1}{ }^{-1}\right\}$

" $\Leftarrow "$ : Let $x, y \in \mathrm{G}_{1}$ with $x \leqslant y$. By Proposition 3.6, we have $\left\{\mathrm{f}(w): w \in x \cdot \mathrm{C}_{1}{ }^{-1}\right\} \subseteq\left\{\mathrm{f}(w): w \in \mathrm{y} \cdot \mathrm{C}_{1}{ }^{-1}\right\}$. Thus $\mathrm{f}(\mathrm{x})=\bigvee\left\{\mathrm{f}(w): w \in x \cdot \mathrm{C}_{1}{ }^{-1}\right\} \leqslant \mathrm{f}(\mathrm{y})=\bigvee\left\{\mathrm{f}(w): w \in \mathrm{y} \cdot \mathrm{C}_{1}{ }^{-1}\right\}$ and so, $\mathrm{f}$ is monotone.

Let $\mathrm{U} \in \beta\left(\mathrm{G}_{2}\right)$ and $x \in \mathrm{f}^{-1}(\mathrm{U})$. By Proposition 3.11, $x \cdot \mathrm{C}_{1}{ }^{-1}$ is directed in $\mathrm{G}_{1}$. Since $f$ is monotone, we have $\left\{f(w): w \in x \cdot C_{1}{ }^{-1}\right\}$ is directed in $G_{2}$. Hence $y^{-1} \cdot\left\{f(w): w \in x \cdot C_{1}{ }^{-1}\right\}$ is directed and $V\left(y^{-1} \cdot\left\{f(w): w \in x \cdot C_{1}^{-1}\right\}\right)=y^{-1} \cdot f(x)$ by Proposition 2.5. Because $f(x) \in U$, there exists $y \in U$ such that $f(x) \in y \cdot C_{2} \subseteq U$ by Proposition 4.3 (1). So $y^{-1} \cdot f(x) \in C_{2}$. Thus $\left(y^{-1} \cdot\left\{f(w): w \in x \cdot C_{1}{ }^{-1}\right\}\right) \cap C_{2} \neq \emptyset$ by Proposition 3.12. This means that there exists $w \in x \cdot C_{1}{ }^{-1}$ such that $y^{-1} \cdot f(w) \in C_{2}$. Hence $x \in w \cdot C_{1}$ and $\mathrm{f}(w) \in \mathrm{y} \cdot \mathrm{C}_{2} \subseteq \mathrm{U}$. Now we will show that $w \cdot \mathrm{C}_{1} \subseteq \mathrm{f}^{-1}(\mathrm{U})$. Let $z \in w \cdot \mathrm{C}_{1}$, i.e., $w \in z \cdot \mathrm{C}_{1}^{-1}$. Then $f(w) \leqslant f(z)$ for $f(z)=\bigvee\left\{f(w): w \in z \cdot C_{1}{ }^{-1}\right\}$. By Proposition 3.5, we have $y \cdot C_{2}$ is an upper set. Thus $\mathrm{f}(z) \in \mathrm{y} \cdot \mathrm{C}_{2} \subseteq \mathrm{U}$ and $z \in \mathrm{f}^{-1}(\mathrm{U})$. Hence $w \cdot \mathrm{C}_{1} \subseteq \mathrm{f}^{-1}(\mathrm{U})$. Since $x \in w \cdot \mathrm{C}_{1}$ and $w \cdot \mathrm{C}_{1} \in \beta\left(\mathrm{G}_{1}\right)$, we have $f^{-1}(U) \in \beta\left(G_{1}\right)$ by Proposition 4.10. Therefore $f$ is continuous.

Proposition 4.12. Let $\mathrm{G}$ be an l-group with the strong-positive cone $\mathrm{C}$. The sets of the form $\mathrm{x} \cdot \mathrm{C}^{-1}, \mathrm{x} \in \mathrm{G}$, constitute a basis of a topology on $\mathrm{G}$ (we call this topology the $\mathrm{C}^{-1}$-topology).

Proof. Let $x \in G$ and $y \in x \cdot C$. Then there exists $a \in C$ such that $y=x \cdot a$. Thus $x=y \cdot a^{-1} \in y \cdot C^{-1} \subseteq$ $\bigcup_{u \in G}\left(u \cdot C^{-1}\right)$. Therefore $G \subseteq \bigcup_{x \in G}\left(x \cdot C^{-1}\right) \subseteq G$ and $G=\bigcup_{x \in G}\left(x \cdot C^{-1}\right)$. Let $x, y \in G$. Since $G$ is an l-group, we conclude that $x \wedge y$ exists and $(x \wedge y) \cdot C^{-1} \subseteq x \cdot C^{-1}$ and $(x \wedge y) \cdot C^{-1} \subseteq y \cdot C^{-1}$ by Proposition 3.6. Hence the family $\left\{x \cdot \mathrm{C}^{-1}: x \in \mathrm{G}\right\}$ constitutes a basis of a topology on $\mathrm{G}$ by Proposition 2.8.

If $G=(G, \leqslant, \cdot)$ is an l-group with the positive cone $P$, the strong-positive cone $C$ and $G^{o p}=(G, \geqslant, \cdot)$ denotes the dual of $G$, then in $G^{\text {op }}$ the positive cone is $P^{-1}$ and the strong-positive cone is $C^{-1}$. So the $C^{-1}$-topology of $G$ is equivalent to the $C$-topology of $G^{o p}$. Thus we denote the $C^{-1}$-topology of $G$ by $\beta\left(G^{o p}\right)$. Moreover, if $G$ is a continuous l-group, then $G^{o p}$ is also a continuous l-group. So we have the following properties:

Proposition 4.13. Let $\mathrm{G}$ be an l-group with the strong-positive cone $\mathrm{C} . \mathrm{U}$ is an open set in the $\mathrm{C}^{-1}$-topology if and only if $\mathrm{U}=\mathrm{U} \cdot \mathrm{C}^{-1}$.

Proposition 4.14. Let $\mathrm{G}_{1}, \mathrm{G}_{2}$ be continuous l-groups with the strong-positive cones $\mathrm{C}_{1}, \mathrm{C}_{2}$, respectively. A function $f: G_{1} \rightarrow G_{2}$ is continuous with respect to the $C^{-1}$-topologies of $G_{1}$ and $G_{2}$ if and only if for any $x \in G_{1}$, $f(x)=\bigwedge\left\{f(w): w \in x \cdot C_{1}\right\}$. 
Definition 4.15. Let $\mathrm{G}$ be an l-group with the strong-positive cone $\mathrm{C}$. Then the common refinement of the C-topology and the $\mathrm{C}^{-1}$-topology is called the bi-C-topology.

Obviously, the bi-C-topology has a basis $\left\{U \cap V: U \in \beta(G), V \in \beta\left(G^{o p}\right)\right\}$, where $\beta(G)$ denotes the C-topology of $G$ and $\beta\left(G^{o p}\right)$ denotes the $C^{-1}$-topology of $G$.

Corollary 4.16. Let $\mathrm{G}_{1}, \mathrm{G}_{2}$ be continuous l-groups with the strong-positive cones $\mathrm{C}_{1}, \mathrm{C}_{2}$, respectively. If a function $\mathrm{f}: \mathrm{G}_{1} \rightarrow \mathrm{G}_{2}$ satisfies: for any $\mathrm{x} \in \mathrm{G}_{1}, \mathrm{f}(\mathrm{x})=\bigvee\left\{\mathrm{f}(w): w \in \mathrm{x} \cdot \mathrm{C}_{1}{ }^{-1}\right\}=\bigwedge\left\{\mathrm{f}(w): w \in x \cdot \mathrm{C}_{1}\right\}$, then $\mathrm{f}$ is continuous with respect to the bi-C-topologies of $\mathrm{G}_{1}$ and $\mathrm{G}_{2}$.

Proof. This result can be proved by Proposition 4.11 and Proposition 4.14 .

Remark 4.17. In an l-group $G$, a basis of the bi-C-topology is $\left\{U \cap V: U \in \beta(G), V \in \beta\left(G^{\text {op }}\right)\right\}$, where $\beta(G)$ denotes the C-topology of $G$. So a basis of the bi-C-topology of $G^{\text {op }}$ is $\left\{U \cap V: U \in \beta\left(G^{\text {op }}\right), V \in \beta(G)\right\}$, where $\beta(G)$ denotes the $C$-topology of $G$. Therefore, open sets in the bi-C-topology of $G$ and open sets in the bi-C-topology of $\mathrm{G}^{\text {op }}$ are exactly the same.

Thus we have the following corollary.

Corollary 4.18. Let $\mathrm{G}_{1}, \mathrm{G}_{2}$ be continuous l-groups with the strong-positive cones $\mathrm{C}_{1}, \mathrm{C}_{2}$, respectively. If a function $\mathrm{f}: \mathrm{G}_{1} \rightarrow \mathrm{G}_{2}$ satisfies: for any $\mathrm{x} \in \mathrm{G}_{1}, \mathrm{f}(\mathrm{x})=\bigwedge\left\{\mathrm{f}(w): w \in \mathrm{x} \cdot \mathrm{C}_{1}{ }^{-1}\right\}=\bigvee\left\{\mathrm{f}(w): w \in \mathrm{x} \cdot \mathrm{C}_{1}\right\}$, then $\mathrm{f}$ is continuous with respect to the bi-C-topologies of $\mathrm{G}_{1}$ and $\mathrm{G}_{2}$.

Proposition 4.19. Let $\mathrm{G}$ be a continuous l-group with the strong-positive cone $\mathrm{C}$. Then the mapping

$$
f: G \times G \rightarrow G, \quad(x, y) \mapsto x \cdot y
$$

is continuous with respect to the $\mathrm{C}$-topologies and the bi-C-topologies of $\mathrm{G} \times \mathrm{G}$ and $\mathrm{G}$.

Proof. Let $(x, y) \in G \times G$. Because $\bigvee\left(x \cdot C^{-1}\right)=x$ and $\bigvee\left(y \cdot C^{-1}\right)=y$ by Proposition 3.11, we have $\bigvee\left(x \cdot C^{-1} \cdot y \cdot C^{-1}\right)=\bigvee\left(x \cdot C^{-1}\right) \cdot \bigvee\left(y \cdot C^{-1}\right)=x \cdot y$ by Proposition 2.6. Since $\left\{f\left(w_{1}, w_{2}\right):\left(w_{1}, w_{2}\right) \in\right.$ $\left.(x, y) \cdot(C \times C)^{-1}\right\}=\left\{w_{1} \cdot w_{2}: w_{1} \in x \cdot C^{-1}, w_{2} \in y \cdot C^{-1}\right\}=x \cdot C^{-1} \cdot y \cdot C^{-1}$, we conclude that $\bigvee\left\{f\left(w_{1}, w_{2}\right):\right.$ $\left.\left(w_{1}, w_{2}\right) \in x \cdot C^{-1}, w_{2} \in y \cdot C^{-1}\right\}=x \cdot y=f(x, y)$. Therefore $f$ is a continuous map with respect to the C-topologies of $\mathrm{G} \times \mathrm{G}$ and $\mathrm{G}$ by Proposition 4.11.

Because $\Lambda(x \cdot C)=x$ and $\bigwedge(y \cdot C)=y$ by Proposition 3.11, we have $\bigwedge(x \cdot C \cdot y \cdot C)=\bigwedge(x \cdot C) \cdot \bigwedge(y \cdot C)=$ $x \cdot y$ by Proposition 2.6. Since $\left\{f\left(w_{1}, w_{2}\right):\left(w_{1}, w_{2}\right) \in(x, y) \cdot C \times C\right\}=\left\{w_{1} \cdot w_{2}: w_{1} \in x \cdot C, w_{2} \in y \cdot C\right\}=$ $x \cdot C \cdot y \cdot C$, we have $\bigwedge\left\{f\left(w_{1}, w_{2}\right):\left(w_{1}, w_{2}\right) \in x \cdot C, w_{2} \in y \cdot C\right\}=x \cdot y=f(x, y)$. Therefore $f$ is a continuous map with respect to the bi-C-topologies of $\mathrm{G} \times \mathrm{G}$ and $\mathrm{G}$ by Corollary 4.16 .

Proposition 4.20. Let $\mathrm{G}$ be a continuous l-group with the strong-positive cone $\mathrm{C}$. Then the mapping

$$
f: G \rightarrow G, \quad x \mapsto x^{-1}
$$

is continuous with respect to the bi-C-topology.

Proof. Let $x \in G$. Then $\bigwedge\left\{f(w): w \in x \cdot C^{-1}\right\}=\bigwedge\left\{w^{-1}: w \in x \cdot C^{-1}\right\}=\bigwedge\left(x^{-1} \cdot C\right)=x^{-1}=f(x)$. Similarly, $\bigvee\{f(w): w \in x \cdot C\}=f(x)$. Hence $f$ is continuous with respect to the bi-C-topology by Corollary 4.18.

Lemma 4.21 ([13]). Let $\mathrm{L}$ be a lattice and $\mathrm{A}, \mathrm{B} \subseteq \mathrm{L}$. If $\bigvee \mathrm{A}, \bigvee \mathrm{B}$ exist in $\mathrm{L}$, then $\bigvee\{\mathrm{a} \vee \mathrm{b}: \mathrm{a} \in \mathrm{A}, \mathrm{b} \in \mathrm{B}\}=$ $(\bigvee A) \vee(\bigvee B)$. Dually, if $\wedge A, \wedge B$ exist in $L$, then $\wedge\{a \wedge b: a \in A, b \in B\}=(\wedge A) \wedge(\wedge B)$.

Lemma 4.22 ([18]). Let $\mathrm{P}$ be a continuous poset and $\mathrm{D}_{1}, \mathrm{D}_{2}$ are directed subsets of $\mathrm{P}$. If $\bigvee \mathrm{D}_{1}, \bigvee \mathrm{D}_{2}$ exist in $\mathrm{P}$, then $\bigvee\left\{d_{1} \wedge d_{2}: d_{1} \in D_{1}, d_{2} \in D_{2}\right\}=\left(\bigvee D_{1}\right) \wedge\left(\bigvee D_{2}\right)$. Dually, let $F_{1}, F_{2}$ be filtered subsets of $P$. If $\wedge F_{1}, \wedge F_{2}$ exist in $P$, then $\bigwedge\left\{f_{1} \vee f_{2}: f_{1} \in F_{1}, f_{2} \in F_{2}\right\}=\left(\bigwedge F_{1}\right) \vee\left(\bigwedge F_{2}\right)$.

Theorem 4.23. Let $\mathrm{G}$ be a continuous l-group with the bi-C-topology $\tau$. Then $(\mathrm{G}, \cdot, \tau)$ is a topological group and $(\mathrm{G}, \leqslant, \tau)$ is a topological lattice. 
Proof. We have $(G, \cdot \tau)$ is a topological group by Proposition 4.19 and Proposition 4.20.

Let $(x, y) \in G \times G$. We will show that $f: G \times G \rightarrow G$ by $(x, y) \mapsto x \vee y$ and $g: G \times G \rightarrow G$ by $(x, y) \mapsto x \wedge y$ are continuous with respect to the bi-C-topologies of $G \times G$ and $G$.

Since $\bigvee\left(x \cdot C^{-1}\right)=x$ and $\bigvee\left(y \cdot C^{-1}\right)=y$ by Proposition 3.11 and $\bigvee\left\{f\left(w_{1}, w_{2}\right):\left(w_{1}, w_{2}\right) \in(x, y) \cdot(C \times\right.$ $\left.C)^{-1}\right\}=\bigvee\left\{w_{1} \vee w_{2}: w_{1} \in x \cdot C^{-1}, w_{2} \in y \cdot C^{-1}\right\}$, we conclude that $\bigvee\left\{f\left(w_{1}, w_{2}\right):\left(w_{1}, w_{2}\right) \in(x, y) \cdot(C \times\right.$ $\left.C)^{-1}\right\}=x \vee y=f(x, y)$ by Lemma 4.21. By the continuity of $G, x \cdot C, y \cdot C$ are filtered sets and $\bigwedge(x \cdot C)=x$, $\bigwedge(y \cdot C)=y$. Because $\bigwedge\left\{f\left(w_{1}, w_{2}\right):\left(w_{1}, w_{2}\right) \in(x, y) \cdot C \times C\right\}=\bigwedge\left\{w_{1} \vee w_{2}: w_{1} \in x \cdot C, w_{2} \in y \cdot C\right\}$, we have $\Lambda\left\{f\left(w_{1}, w_{2}\right):\left(w_{1}, w_{2}\right) \in(x, y) \cdot C \times C\right\}=x \vee y=f(x, y)$ by Theorem 3.14 and Lemma 4.22. Hence $f$ is continuous with respect to the bi-C-topologies of $\mathrm{G} \times \mathrm{G}$ and $\mathrm{G}$ by Corollary 4.16.

By Proposition 3.11, $x \cdot C^{-1}, y \cdot C^{-1}$ are directed sets and $\bigvee\left(x \cdot C^{-1}\right)=x, V\left(y \cdot C^{-1}\right)=y$. Because $\bigvee\left\{g\left(w_{1}, w_{2}\right):\left(w_{1}, w_{2}\right) \in(x, y) \cdot(C \times C)^{-1}\right\}=\bigvee\left\{w_{1} \wedge w_{2}: w_{1} \in x \cdot C^{-1}, w_{2} \in y \cdot C^{-1}\right\}$, we have $\bigvee\left\{g\left(w_{1}, w_{2}\right):\right.$ $\left.\left(w_{1}, w_{2}\right) \in(x, y) \cdot(C \times C)^{-1}\right\}=x \wedge y=g(x, y)$ by Theorem 3.14 and Lemma 4.22. Since $\bigwedge(x \cdot C)=x$ and $\Lambda(y \cdot C)=y$ by the continuity of $G$ and $\bigwedge\left\{g\left(w_{1}, w_{2}\right):\left(w_{1}, w_{2}\right) \in(x, y) \cdot C \times C\right\}=\bigwedge\left\{w_{1} \wedge w_{2}: w_{1} \in\right.$ $\left.x \cdot C, w_{2} \in y \cdot C\right\}$, we conclude that $\Lambda\left\{g\left(w_{1}, w_{2}\right):\left(w_{1}, w_{2}\right) \in(x, y) \cdot C \times C\right\}=x \wedge y=g(x, y)$ by Lemma 4.21. Hence $g$ is continuous with respect to the bi-C-topologies of $G \times G$ and $G$ by Corollary 4.16. Therefore $(G, \leqslant, \tau)$ is a topological lattice.

\section{Acknowledgment}

We would like to thank the anonymous referees for their careful reading and valuable comments which have improved the quality of this paper.

This work is supported by National Natural Science Foundation of China (No. 11771134).

\section{References}

[1] M. A. Abdelgawad, A domain-theoretic model of nominally-typed object-oriented programming, Electron. Notes Theor. Comput. Sci., 301 (2014), 3-19. 1

[2] M. A. Abdelgawad, Domain theory for modeling oop: A summary, arXive, 2014 (2014), 15 pages. 1

[3] R. M. Amadio, P.-L. Curien, Domains and lambda-calculi, Cambridge University Press, Cambridge, (1998). 1

[4] A. Arhangel'skii, M. Tkachenko, Topological Groups and Related Structures, Atlantis Press/World scintific, Paris, (2008). 2.9

[5] R. N. Ball, Topological lattice-ordered groups, Pacific J. Math., 83 (1979), 1-26. 1

[6] R. N. Ball, Convergence and cauchy structures on lattice ordered groups, Trans. Amer. Math. Soc., 259 (1980), $357-392$. 1

[7] R. N. Ball, Truncated abelian lattice-ordered groups I: The pointed (Yosida) representation, Topology Appl., 162 (2014), 43-65. 1

[8] R. N. Ball, A. W. Hager, Epi-topology and epi-convergence for archimedean lattice-ordered groups with unit, Appl. Categ. Structures, 15 (2007), 81-107. 1

[9] G. Birkhoff, Lattice-ordered groups, Ann. of Math., 43 (1942), 298-331. 1

[10] G. Birkhoff, Lattice theory, American Mathematical Society, Providence, (1967). 1

[11] J. L. Castiglioni, H. J. San Martín, The left adjoint of spec from a category of lattice-ordered groups, J. Appl. Log., 15 (2016), 1-15. 1

[12] A. H. Clifford, Partially ordered abelian groups, Ann. of Math., 41 (1940), 465-473. 1

[13] B. A. Davey, H. A. Priestley, Introduction to lattices and order, Second edition, Cambridge university press, New York, (2002). 4.5, 4.21

[14] A. Edalat, Domains for computation in mathematics, physics and exact real arithmetic, Bull. Symbolic Logic, 3 (1997), 401-452. 1

[15] L. Fuchs, Partially ordered algebraic systems, Dover publication, Inc., New York, (1963). 1, 2.2, 2.5, $2.6,2.7$

[16] L. Fuchs, Riesz vector spaces and Riesz algebras, Séminaire Dubreil. Algèbre et théorie des nombres, 19 (1965/66), 1-9. 1

[17] G. Gierz, K. H. Hofmann, K. Keimel, J. D. Lawson, M. Mislove, D. S. Scott, Continuous Lattices and Domains, Cambridge University Press, Cambridge, (2003). 1, 2.3

[18] J. Goubault-Larrecq, Non-Hausdorff topology and domain theory [On the cover: Selected topics in point-set topology], Cambridge University Press, Cambridge, (2013). 1, 2.3, 4.7, 4.8, 4.22

[19] G. Grätzer, General lattice theory, Second edition, Birkhäuser Verlag, Basel, Boston, Berlin, (2003). 2.10

[20] L. Guo, Q. Li, The Categorical Equivalence Between Algebraic Domains and F-Augmented Closure Spaces, Order, 32 (2015), 101-116. 1 
[21] I. Gusić, A topology on lattice ordered groups, Proc. Amer. Math. Soc., 126 (1998), 2593-2597. 1

[22] L. Hong, Locally solid topological lattice-ordered groups, Arch. Math. (Brno), 51 (2015), 107-128. 1

[23] X. Jia, A. Jung, H. Kou, Q. Li, H. Zhao, All cartesian closed categories of quasicontinuous domains consist of domains, Theoret. Comput. Sci., 594 (2015), 143-150. 1

[24] V. M. Kopytov, N. Y. Medvedev, The theory of lattice-ordered groups, Springer Science and Business Media, Dordrecht, (2013). 1, 2.2, 2.4, 2.5, 2.7

[25] L. Li, Q. Jin, p-Topologicalness and p-regularity for lattice-valued convergence spaces, Fuzzy Sets and Systems, 238 (2014), 26-45. 1

[26] L. Li, Q. Jin, G. Meng, K. Hu, The lower and upper p-topological (p-regular) modifications for lattice-valued convergence space, Fuzzy Sets and Systems, 282 (2016), 47-61. 1

[27] J. R. Munkres, Topology, Second edition, Prentice Hall, Inc., Upper Saddle River, NJ, (2000). 2.8, 4.10

[28] G. D. Plotkin, Post-graduate lecture notes in advanced domain theory, Dept. of Computer Science, Univ. of Edinburgh, (1981). 1

[29] J. C. Reynolds, Theories of programming languages, Cambridge University Press, Cambridge, (2009). 1

[30] L. A. Ricarte, S. Romaguera, A domain-theoretic approach to fuzzy metric spaces, Topology Appl., 163 (2014), $149-159$. 1

[31] D. S. Scott, Outline of a mathematical theory of computation, Oxford University Computing Laboratory, Programming Research Group, Oxford, (1970). 1

[32] D. S. Scott, Continuous lattices, Lecture Notes in Mathematics, Springer, Berlin, (1972). 1

[33] D. S. Scott, Data types as lattices, Lecture Notes in Mathematics, Springer, Berlin, (1975). 1

[34] D. S. Scott, A type-theoretical alternative to iswim, cuch, owhy, Theoret. Comput. Sci., 121 (1993), 411-440. 1

[35] X. Xi, Q. He, L. Yang, On the largest cartesian closed category of stable domains, Theoret. Comput. Sci., 669 (2017), 22-32. 1

[36] Y. C. Yang, l-groups and bézout domains, PHD thesis, Von der Fakultat Math. und Physik der Universitat Stuttgart, (2006). 1

[37] Y. C. Yang, W. Rump, Bézout domains with nonzero unit radical, Comm. Algebra, 38 (2010), 1084-1092. 1

[38] H. Zhao, H. Kou, Tw as a stable universal domain, Electron. Notes Theor. Comput. Sci., 301 (2014), 189-202. 1 